\title{
ASYMPTOTIC BOUNDS FOR PRECISE LARGE DEVIATIONS IN A COMPOUND RISK MODEL UNDER DEPENDENCE STRUCTURES
}

\author{
Qingwu GaO*, XiJun LiU And Chunhong Chai
}

Abstract. In the paper, we consider a compound risk model, where all the claim sizes satisfy a dependence structure, and the accident inter-arrival time and the claim-number of the subsequent accident satisfy another dependence structure described by a conditional tail probability of the inter-arrival time given the subsequent claim-number. We obtain the asymptotic lower and upper bounds for the precise large deviations of the aggregate claims, with a feature that the asymptotic bounds hold uniformly for all $x$ in an infinite $t$-interval.

Mathematics subject classification (2010): Primary 60F10; Secondary 62P05, 91B30.

Keywords and phrases: Asymptotics, precise large deviation, compound risk model, dependence structure.

\section{REFERENCES}

[1] N. Bingham, C. Goldie And J. Teugels, Regular variation, Cambridge University Press, Cambridge, 1987.

[2] Y. Chen AND K. Yuen, Precise large deviations of aggregate claims in a size-dependent renewal risk model, Insur. Math. Econ., 51 (2): 457-461, 2012.

[3] Y. Chen, K. YUen AND K. NG, Precise large deviations of random sums in presence of negative dependence and consistent variation, Methodol. Comput. Appl. Probab., 13 (4): 821-833, 2011.

[4] D. Cline AND T. Hsing, Large deviation probabilities for sums and maxima of random variables with heavy or subexponential tails, Texas A\&M University, 1991.

[5] D. DENISOV, S. Foss, AND D. KorshunOv, Tail asymptotics for the supremum of a random walk when the mean is not finite, Queu. Syst., 46: 15-33, 2004.

[6] P. Embrechts, C. KlÜPpelberg And T. Mikosch, Modelling extremal events for insurance and finance, Springer, Berlin, 1997.

[7] K. WANG AND L. CHEN, Precise large deviations for the aggregate claims in a dependent compound renewal risk model, J. Inequal. Appl. 257, 2019, doi:10.1186/s13660-019-2209-1.

[8] J. GeluK AND Q. TANG, Asymptotic tail probabilities of sums of dependent subexponential random variables, J. Theoret. Probab., 22 (4): 871-882, 2009.

[9] W. HE, D. Cheng AND Y. WANG, Asymptotic lower bounds of precise large deviations with nonnegative and dependent random variables, Statist. Probab. Lett., 83: 331-338, 2013.

[10] T. JIANG, S. CUI AND R. MING, Large deviations for the stochastic present value of aggregate claims in the renewal risk model, Statist. Probab. Lett., 101: 83-91, 2015.

[11] J. LI, Q. TANG AND R. WU, Subexponential tails of discounted aggregate claims in a time-dependent renewal risk model, Adv. Appl. Probab., 42: 1126-1146, 2010.

[12] L. LiU, Precise large deviations for dependent variables with heavy tails, Statist. Probab. Lett., 79 (9): 1290-1298, 2009.

[13] X. LIU, Q. GAO AND M. LIU, Large deviations for the stochastic present value of aggregate claims in the nonstandard compound renewal risk model with widely upper orthant dependent claims, Commun. Stat.-Theor. M., 49 (13): 3073-3093, 2020.

[14] X. LiU, Q. GAO AND Y. WANG, A note on a dependent risk model with constant interest rate, Statist. Probab. Lett., 82: 707-712, 2012. 
[15] X. Liu, C. Yu And Q. GaO, Precise large deviations of aggregate claim amount in a dependent renewal risk model, Comm. Statist. Theory Methods, 46: 2354-2363, 2017.

[16] F. Loukiss As, Precise large deviations for long-tailed distributions, J. Theoret. Probab., 25 (4): $913-$ 924, 2012.

[17] D. LU, L. Song AND Y. XU, Precise large deviations for sums of independent random variables with consistently varying tails, Comm. Statist. Theory Methods., 43: 28-43, 2014.

[18] J. KoČEtova, R. LeIPUS AND J. ŠIAUlys, A property of the renewal counting process with application to the finite-time ruin probability, Lith. Math. J., 49: 55-61, 2009.

[19] D. Konstantinides AND F. LOUKISSAS, Precise large deviations for consistently varying-tailed distributions in the compound renewal risk model, Lith. Math. J., 50 (4): 391-400, 2010.

[20] D. Konstantinides AND F. LOUKISS As, Precise large deviations for sums of negatively dependent random variables with common long-tailed distributions, Comm. Statist. Theory Methods, 40: 36633671, 2011.

[21] C. KlÜPPElberg AND T. MikosCh, Large deviations of heavy-tailed random sums with applications in insurance and finance, J. Appl. Probab., 34: 293-308, 1997.

[22] R. KAAS AND Q. TANG, A large deviation result for aggregate claims with dependent claim occurencies, Insur. Math. Econ., 36: 251-259, 2005.

[23] T. Mikosch ANd A. NAGAEv, Large deviations of heavy-tailed sums with applications in insurance, Extremes, 1: 81-110, 1998.

[24] K. NG, Q. TANG, J. YAn AND H. YANG, Precise large deviations for sums of random variables with consistently varying tails, J. Appl. Probab., 41 (1): 93-107, 2004.

[25] D. QIu And P. Chen, Complete and Complete Moment Convergence for Weighted Sums of Widely Orthant Dependent Random Variables, Acta. Math. Sin., English Series 30 (9): 1539-1548, 2014.

[26] Q. TANG, Insensitivity to negative dependence of the asymptotic behavior of precise large deviations, Electron. J. Probab., 11: 107-120, 2006.

[27] Q. TANG, C. SU, T. JIANG AND J. ZHANG, Large deviations for heavy-tailed random sums in compound renewal model, Statist. Probab. Lett., 52 (1): 91-100, 2001.

[28] Q. TAng And G. Tsitsiashvili, Precise estimates for the ruin probability in finite horizon in a discrete-time model with heavy-tailed insurance and financial risks, Stoch. Proc. Appl., 108: 299325, 2003.

[29] Y. WANG AND D. CHENG, Basic renewal theorems for a random walks with widely dependent increments, J. Math. Anal. Appl., 384: 597-606, 2011.

[30] S. WANG AND W. WANG, Precise large deviations for sums of random variables with consistently varying tails in multi-risk models, J. Appl. Probab., 44 (4): 889-900, 2007.

[31] K. WANG, Y. WANG AND Q. GAO, Uniform asymptotics for the finite-time ruin probability of a dependent risk model with a constant interest rate, Methodol. Comput. Appl. Proba., 15: 109-124, 2013.

[32] K. Wang, Y. YAng And J. Lin, Precise large deviations for widely orthant dependent random variables with dominatedly varying tails, Front. Math. China, 7 (5): 919-932, 2012.

[33] X. WAng, C. XU, T. Hu, A. Volodin AND S. Hu, On complete convergence for widely orthantdependent random variables and its applications in nonparametric regression models, TEST 23: 607$629,2014$.

[34] X. WANG AND S. Hu, The consistency of the nearest neighbor estimator of the density function based on WOD samples, J. Math. Anal. Appl. 429 (1): 497-512, 2015.

[35] M. XIAO, S. CUI, R. MING AND T. JIANG, Large deviations for the stochastic present value of aggregate net claims with infinite variance in the renewal risk model and its application in risk management, Cluster Computing, 21 (1): 997-1007, 2018.

[36] M. Xi, R. WANG, Z. Cheng AND X. WANG, Some convergence properties for partial sums of widely orthant dependent random variables and their statistical applications, Stat. Papers, 2018, https://doi.org/10.1007/s00362-018-0996-y.

[37] Y. YAng, L. RemigiJus AND J. ŠIAUlys, Precise large deviations for compound random sums in the presence of dependence structures, Comput. Math. App., 64: 2074-2083, 2012.

[38] Y. YANG AND K. WANG, Precise large deviations for dependent random variables with applications to the compound renewal risk model, Rocky Mountain J. Math., 43 (4): 1395-1414, 2013. 